\title{
AN ACHIEVABLE PERFORMANCE UPPER BOUND FOR DISCRETE MULTITONE EQUALIZATION
}

\author{
Ming Ding, Zukang Shen, and Brian L. Evans
}

\author{
Embedded Signal Processing Lab, Wireless Networking and Commmunications Group \\ The University of Texas at Austin, Austin, TX 78712 USA \\ E-mail: mding@bandspeed.com, \{shen,bevans\}@ece.utexas.edu
}

\begin{abstract}
Discrete multitone is a popular discrete Fourier transform based implementation of multicarrier modulation for wireline communications. This paper examines the performance of a complex-tap filter bank structure for channel equalization in discrete multitone modulation systems. The structure under study passes the received signal through a number of logical paths. Each path takes care of one data carrying subcarrier. More specifically, each path cascades a finite impulse response frequency selective equalizer and a Goertzel filter computing a single point discrete Fourier transform. The delay on each logical path is individually optimized for best performance. In the presented simulations testing achievable bit rate on an asymmetric digital subscriber line transceiver, the filter bank appears to benchmark the bit rate performance among existing discrete multitone equalization methods. An iterative training procedure, which depends on the second-order statistics of the input and output sequences, is proposed to show the achievablity of performance.
\end{abstract}

\section{INTRODUCTION}

Multicarrier modulation (MCM) is a favorable choice over single carrier modulation in wide-band communications with frequency selectivity in transmission bands. MCM techniques such as orthogonal frequency division multiplexing (OFDM) and discrete multi-tone (DMT) modulation have been receiving increasing attention in the literature, and have been incorporated into numerous standards. MCM divides a transmission band into orthogonal subchannels. In a wireline broadband communication system, some frequency discontinuities of transmission band normally exist due to various line impairments. MCM is attractive due to the ease with

This research was supported by The State of Texas Advanced Technology Program under project 003658-06142001. which it can combat channel dispersion without implementation of sharp band-stop filters as in the case of single carrier transmission. In addition to the benefits of MCM, DMT has an extra ability to perform dynamic bit loading, which has the potential to exploit the available bandwidth fully. In broadband wireline communications, DMT modulation is standardized for asymmetric digital subscribe line (ADSL) and very-highspeed digital subscriber line (VDSL) modems. Maximizing the bit rate is the ultimate goal for such systems.

Equalization in an DMT system gains help from a mechanism called a cyclic prefix $(\mathrm{CP})$, which is typically inserted between successive symbols. When the channel order does not exceed the CP length $\nu$, equalization is easily performed in frequency domain via a one-tap complex scalar for each subchannel. But in wireline communications, a transmission line usually has much longer memory than the value of $\nu$ defined by standards. A common practice is to add an finite impulse response (FIR) filter at the front end of a receiver to shorten the channel impulse response to be at most $\nu+1$ samples in length.

Accordingly, the conventional DMT equalizer structure consists of a cascade of a single-FIR time-domain equalizer (TEQ), a fast Fourier transform (FFT), and a single-tap frequency-domain equalizer (FEQ) per tone. Different DMT TEQ design methods optimize FIR coefficients based on training data under different criteria. Minimum mean squared error (MMSE) design $[1,2]$ minimizes the mean square error between the output of the physical path consisting of the channel and FIR filter and the output of a virtual path consisting of a transmission delay $\Delta$ and a target impulse response. The maximum shortening SNR (MSSNR) [3] method attempts to minimize intersymbol interference (ISI) in the time domain. The MSSNR method maximizes the ratio of the energy of the effective channel impulse response inside a target window of $\nu+1$ samples to that outside the target window. Alternate objective functions include maximizing the ratio of the energy inside 
the target window to the total energy [4], and minimizing (maximizing) the energy outside (inside) the target window while holding the energy inside (outside) the target window fixed. The Minimum-ISI (Min-ISI) method generalizes the MSSNR method by weighting the ISI in the frequency domain [5,6], e.g., to place the ISI in unused and more noisy subchannels.

The traditional TEQ-FEQ structure equalizes all subchannels in a combined fashion, which may limit bit rate performance. This time domain approach appears against the original divide-and conquer idea of DMT. At least three alternate equalizer structures have been proposed with improved performance. The dualFIR TEQ [7] uses a standard single-FIR TEQ design algorithm to achieve good bit rate over the entire transmission bandwidth, and uses a second-FIR TEQ design algorithm to improve the bit rate over a subset of subcarriers. The per-tone equalizer [8] essentially moves the single-FIR TEQ into the FEQ, which converts the FEQ into a linear combiner for each subcarrier. A third alternative structure is a TEQ filter bank [9], in which a different FIR TEQ is designed for each tone. The FFT becomes a bank of Goertzel filters and a singletap FEQ is also used.

To study the bit rate performance upper bound in a DMT modulated system, we construct a new equalization structure motivated by TEQ filter bank and per tone equalizer. In this model we move all FEQ operations to the time domain and combine this with the TEQ to obtain a multi-tap complex-valued FIR for each tone. This structure provides time domain equalizer designers the most freedom. The design freedom is equivalent to the per-tone structure for frequency domain equalizers. Furthermore, it can perform delay optimization on each individual path, which per-tone cannot do if sliding FFT or modified single FFT structure is applied. This constructed complex-tap time domain equalizer filter bank is meant to provide an upper bound of bit rate performance theoretically. However, it is also implementable in TI's TMS320C6000 digital signal processor (DSP), which has a clock faster than $200 \mathrm{MHz}$. A TEQ filter bank design procedure, which depends on the second-order statistics of the input and output sequences, is provided. Particularly, in the proposed training algorithm, channel estimation is not required as it is in many other equalization schemes $[1]-[6]$.

\section{COMPLEX-TAP FILTER BANK EQUALIZER STRUCTURE}

Fig. 1 depicts a traditional DMT system with a TEQFEQ equalization structure at the receiver. Information bearing bits are divided into a set of independent data transmission subchannels. Signals are QAMmodulated on each subchannel. An inverse FFT (IFFT)

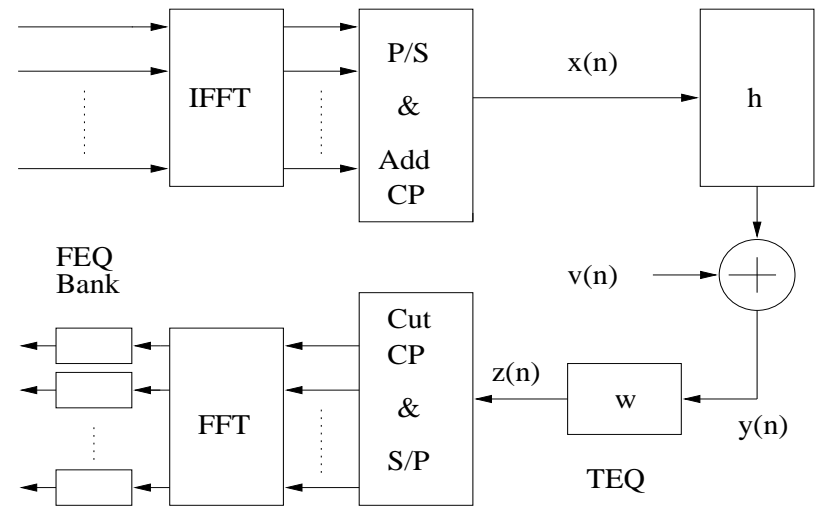

Figure 1: System model. (I)FFT: (inverse) fast Fourier transform, P/S: parallel to serial, S/P: serial to parallel.

converts signal on each subchannel (which acts as one of the frequency components) into a time-domain signal. The input to the channel is made to appear circular by adding a cyclic prefix to the start of each data block. The cyclic prefix is a copy of the last $\nu$ samples of each block. We use the notation $x(n), h(n)$, and $y(n)=\sum_{i=0}^{L_{h}-1} h(i) * x(n-i)+v(n)$ to denote the channel input, channel impulse response, and noiseadded channel output, respectively, where $L_{h}$ denotes the channel length.

If the CP is at least as long as the channel, then the CP-removed channel output is equivalent to a circular convolution of channel and data. After the FFT converts the received data to the frequency domain, the signals can then be equalized by a bank of complex scalers, referred to as FEQs. If the channel is longer than $\nu+1$ samples, a TEQ is needed to shorten the channel. We have pointed out that a single TEQ is far from optimum in terms of bit rate performance. We start from a TEQ filter bank structure to construct the bit rate maximizing structure.

At a DMT receiver with the TEQ filter bank structure (TEQFB) [9], the channel output goes through a bank of TEQs. The FFT block can be implemented as a bank of Goertzel filters with each one computing a single point DFT. There are $N_{c}=256$ possible data carrying subchannels if FFT size $N$ equals 512 .

Fig. 2 shows the proposed complex-tap time domain equalizer filter bank (CTEQFB) structure. Let $t$ indicate the DMT symbol index, $n=0,1, \ldots, N+\nu-1$ indicate the samples within the given symbol, and $\Delta$ be transmission delay of the signal from the transmitter to receiver. The output of the $i$ th TEQ, $z_{i, t}(n)$ is

$$
z_{i, t}(n)=\sum_{\tau=0}^{L_{w}} \mathbf{w}_{i}(\tau) y_{t}(n-\tau)
$$




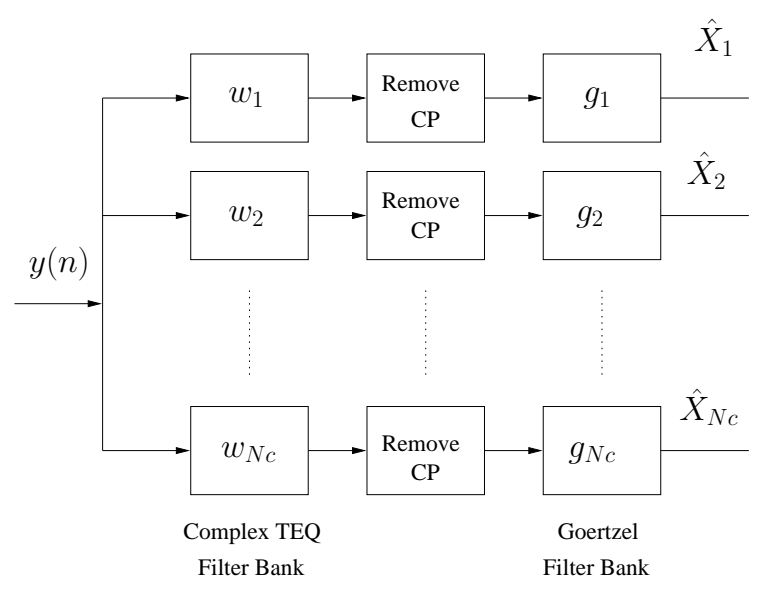

Figure 2: Proposed complex-tap time domain equalizer filter bank for multicarrier modulation with a cyclic prefix (CP).

where $\mathbf{w}_{i}$ denotes the length $L_{w}+1$ column vector of the the TEQ coefficients and $y_{t}(n)=y(n-\Delta)$ is the received sequence with delay reference. For each symbol, only $N$ samples from $Z_{t}(\nu)$ to $Z_{t}(N+\nu-1)$ are used for further processing. The other samples are discarded since they correspond to the heavily ISI-corrupted CP. The $i$ th TEQ operation in the receiver can be written in matrix form as

$$
\begin{aligned}
& \mathbf{z}_{i, t}= \\
& {\left[\begin{array}{cccc}
y_{t}(\nu) & y_{t}(\nu-1) & \ldots & y_{t}\left(\nu-L_{w}\right) \\
y_{t}(\nu+1) & y_{t}(\nu) & \cdots & y_{t}\left(\nu-L_{w}+1\right) \\
\vdots & \vdots & \ddots & \vdots \\
y_{t}(N+\nu-1) & \cdots & \cdots & y_{t}\left(N+\nu-1-L_{w}\right)
\end{array}\right]} \\
& \times\left[\begin{array}{c}
\mathbf{w}_{i}(0) \\
\mathbf{w}_{i}(1) \\
\vdots \\
\mathbf{w}_{i}\left(L_{w}\right)
\end{array}\right]=\mathbf{Y}_{t} \mathbf{w}_{i}
\end{aligned}
$$

where $\mathbf{Y}_{t}$ is Toeplitz matrix which contains the received signal for detection of $t$ th symbol. The output of $i$ th Goertzel filter is

$$
\begin{aligned}
g_{t, i} & =\left[\begin{array}{llll}
W_{N}^{i 0} & W_{N}^{i 1} & \ldots & W_{N}^{i(N-1)}
\end{array}\right] z_{t, i} \\
& =\mathbf{q}_{i}^{H} \mathbf{Y}_{t} \mathbf{w}_{i}
\end{aligned}
$$

where $W_{N}=e^{-j 2 \pi / N}$ is the DFT complex quantity and $\mathbf{q}_{i}^{H}$ is the $i$ th row of DFT matrix. The output of the $i$ th one-tap FEQ (denoted as $\phi_{i}$ ) is then

$$
\begin{aligned}
\hat{X}_{t, i} & =\phi_{i} \times g_{t, i} \\
& =\phi_{i} \mathbf{q}_{i}^{H} \mathbf{Y}_{t} \mathbf{w}_{i}
\end{aligned}
$$

We can move all FEQ operations to the time domain and combine them with the TEQ to obtain a multi-tap complex valued FIR filter for each tone. If we denote the filter for subchannel $i$ as $\tilde{\mathbf{w}}_{i}$, then we have the output of the $i$ th Goertzel filter as

$$
\hat{X}_{t, i}=\mathbf{q}_{i}^{H} \mathbf{Y}_{t} \tilde{\mathbf{w}}_{i}
$$

which is ready for decision making.

In terms of design freedom, we compare the existing four equalization structures. By inspecting (5), we conclude that the conventional TEQ-FEQ structure has the least freedom. It uses only one TEQ w for all subchannels. TEQFB lifts this restriction to have $\mathbf{w}_{i}$ for each used subchannel, but it has a common frequency domain scaler $\phi_{i}$ for each tap of the TEQ. Per-tone equalizer moves the TEQ to the frequency domain and directly optimize on multi-tap FEQ. CTEQFB moves the FEQ into the TEQ. Both of them could have a different scaler $\phi_{i, j}$ for $j$ th tap of the TEQ $\mathbf{w}_{i}$. Thus, the per-tone equalizer and CTEQFB have the most design freedom. The per-tone equalizer is trained in the DFT domain at the symbol rate (e.g. $4 \mathrm{kHz}$ in ADSL). The CTEQFB is trained at the sampling rate (e.g. 2.208 $\mathrm{MHz}$ in ADSL). During data transmission, however, the implementation complexity of CTEQFB is much higher than that of a per-tone equalizer since per-tone equalizer exploits Toeplitz structure of $\mathbf{z}_{i, t}$. However, per-tone equalizer with this efficient implementation relies on a common delay parameter for all subchannels while CTEQFB does not subject to this constraint. Hence, we expect CTEQFB reaches a higher bit rate after delay optimization performed on each single logic path.

\section{COMPLEX-TAP FILTER BANK EQUALIZER TRAINING}

In wireline MCM systems such as ADSL and VDSL, it is desirable that the optimization of each subsystem finally leads to bit rate maximization for a target bit error rate tolerance. The number of bits per symbol for the proposed CTEQFB is

$$
b_{C T E Q F B}=\sum_{i} \log _{2}\left(1+\frac{\operatorname{SNR}\left(w_{i}\right)}{\Gamma}\right)
$$

where $\Gamma$ is the excessive SNR required to reach Shannon capacity. Various SNR models exist in current literature. Most of them adopt an approximate modeling of noise. [10] only considers additional noise, [5] takes ISI into account, and [9] models noise as sum of ISI, additive white Gaussian noise (AWGN), Near End Crosstalk (NEXT) and digital noise floor. The latter is very close to true configuration of impairments, but there are other possible noise sources such as radio frequency interference (RFI), clipping noise and FFT leakage left unattended. 
In order to model noise as accurate as possible, we define SNR at the $i$ th Goertzel filter output as

$$
\mathrm{SNR}_{i}=\frac{E\left[\left|X_{t, i}\right|^{2}\right]}{E\left[\left|X_{t, i}-\hat{X}_{t, i}\right|\right]^{2}}
$$

where $E$ stands for expectation, and $X_{t, i}$ is the frequency domain input on the $i$ th subchannel at $t$ th symbol. In a wireline MCM system, the expected energy $E\left[\left|X_{t, i}\right|^{2}\right]$ is constant across all subchannels during training. Thus, we can write $E\left[\left|X_{t, i}\right|^{2}\right]=S_{x}$. A similar SNR definition can be found in [11]. With this definition, we implicitly include all possible noise sources up to the point before slicer.

It is obvious that maximizing the SNR in each single subchannel also maximizes the bit rate. It turns out the maximization of $\mathrm{SNR}_{i}$ is equivalent to the minimization of

$$
\begin{aligned}
J_{i}= & E\left[\left|X_{t, i}-\hat{X}_{t, i}\right|\right]^{2} \\
= & E\left[\left|X_{t, i}-\hat{X}_{t, i}\right|\left|X_{t, i}-\hat{X}_{t, i}\right|^{*}\right] \\
= & S_{x}-\tilde{\mathbf{w}}_{i}^{H} E\left[X_{t, i} \mathbf{Y}_{t}^{H} \mathbf{q}_{i}\right]-E\left[\mathbf{q}_{i}^{H} \mathbf{Y}_{t} X_{t, i}^{*}\right] \tilde{\mathbf{w}}_{i} \\
& \quad+\tilde{\mathbf{w}}_{i}^{H} E\left[\mathbf{Y}_{t}^{H} \mathbf{q}_{i} \mathbf{q}_{i}^{H} \mathbf{Y}_{t}\right] \tilde{\mathbf{w}}_{i}
\end{aligned}
$$

To minimize $J_{i}$ with respect to $\tilde{\mathbf{w}}_{i}$, we set

$$
\frac{\partial J_{i}}{\partial \tilde{\mathbf{w}}_{i}^{*}}=E\left[\mathbf{Y}_{t}^{H} \mathbf{q}_{i} \mathbf{q}_{i}^{H} \mathbf{Y}_{t}\right] \tilde{\mathbf{w}}_{i}-E\left[\mathbf{Y}_{t}^{H} \mathbf{q}_{i} X_{t, i}\right]=0
$$

Only second-order input-output statistics are required to solve this linear estimation problem. In other words, this approach is not based on channel estimation. Also, blind equalization is feasible provided that we have pre-knowledge of input distributions. However, we have the luxury of training sequence offered in many wireline communications standards. A practical solution to train CTEQFB for a wireline multicarrier receiver is formed as follows:

1. Use training sequence to get time average estimations of all expectation items in (10).

2. Solve the linear equation in (10). $Y_{t}$ terms are observed to be linearly independent between symbols. Time average of $\mathbf{Y}_{t}^{H} \mathbf{q}_{i} \mathbf{q}_{i}^{H} \mathbf{Y}_{t}$ is always full rank. Thus, a unique solution

$$
\tilde{\mathbf{w}}_{i}=\hat{E}\left[\mathbf{Y}_{t}^{H} \mathbf{q}_{i} \mathbf{q}_{i}^{H} \mathbf{Y}_{t}\right]^{-1} \hat{E}\left[\mathbf{Y}_{t}^{H} \mathbf{q}_{i} X_{t, i}\right]
$$

can be obtained.

An iterative solution is provided in Fig. 3 based on a complex valued steepest decent searching method.

Usually we need to perform delay optimization to obtain the highest achievable bit rate out of possible transmission delays. In the conventional TEQ-FEQ structure, optimization ends up with one optimum $\Delta$
1. For loaded subchannel $i$, estimate time average of $\mathbf{A}_{i}=\left(X_{i}^{*} \mathbf{q}_{i}^{H} \mathbf{Y}_{t}\right)^{T}$ and $\mathbf{B}_{i}=$ $\left(\mathbf{q}_{i}^{H} \mathbf{Y}_{t}\right)^{T}\left(\mathbf{q}_{i}^{H} \mathbf{Y}_{t}\right)^{*}$ over a number of blocks

2. Start with non-zero initial guess $\mathbf{w}_{i}^{0}$

3. Choose step size $\mu_{i}$ satisfies $0<\mu_{i}<$ $\frac{1}{2 \times \operatorname{trace}\left(\mathbf{B}_{i}\right)}$

4. Pre-compute $\Lambda_{i}=\left(I-\mu_{i} \mathbf{B}_{i}\right)$ and $\Phi_{i}=\mu_{i} \mathbf{A}_{i}$

5. For $k=1,2, \ldots, \mathbf{w}_{i}^{k+1}=\Lambda_{i} \mathbf{w}_{i}^{k}+\Phi_{i}$

6. When iteration stops, $\mathbf{w}_{i}=\mathbf{w}_{i}^{*}$

Figure 3: Proposed iterative CTEQFB design method

for all frequency components of the signal. To see the true capability a CTEQFB structure has, we can acctually introduce distinct optimum transmission delay $\Delta_{i}$ on path $i$ for signals on subchannel $i$. A better bit rate performance is expected under this delay optimization.

\section{SIMULATIONS}

The simulations compare the bit rate performance of the different equalization structures for a wireline communication system. One typical design for each structure is adopted. The system setup that we consider is the downstream transmission of an ADSL transceiver. According to the G992.1 standard, the IFFT and FFT lengths are 512 and the cyclic prefix length is 32 . We test our designs on 8 typical carrier service area (CSA) loops recommended by Bell Labs [12]. A 5th order high pass IIR filter with passband frequency at 138 $\mathrm{kHz}$ is used to separate the downstream data from the upstream data. The signal power spectral density at the transmitter output is set equal to $-40 \mathrm{dBm} / \mathrm{Hz}$. Channel noise is modeled as an additive white Gaussian noise (AWGN) with $-140 \mathrm{dBm} / \mathrm{Hz}$ power density, NEXT noise from 5 integrated services digital network (ISDN) disturbers, and RFI at seven amplitude modulated (AM) radio carrier frequencies: $540 \mathrm{kHz}, 650$ $\mathrm{kHz}, 680 \mathrm{kHz}, 760 \mathrm{kHz}, 790 \mathrm{kHz}, 840 \mathrm{kHz}$ and 1080 $\mathrm{kHz}$.

Bit allocation on different subcarriers is calculated by

$$
b_{i}=\log _{2}\left(1+\frac{\mathrm{SNR}_{i}}{\Gamma_{\text {sim }}}\right)
$$

where $i$ varies over all data carrying subcarriers, $\mathrm{SNR}_{i}$ is the SNR at the $i$ th subcarrier derived from average 


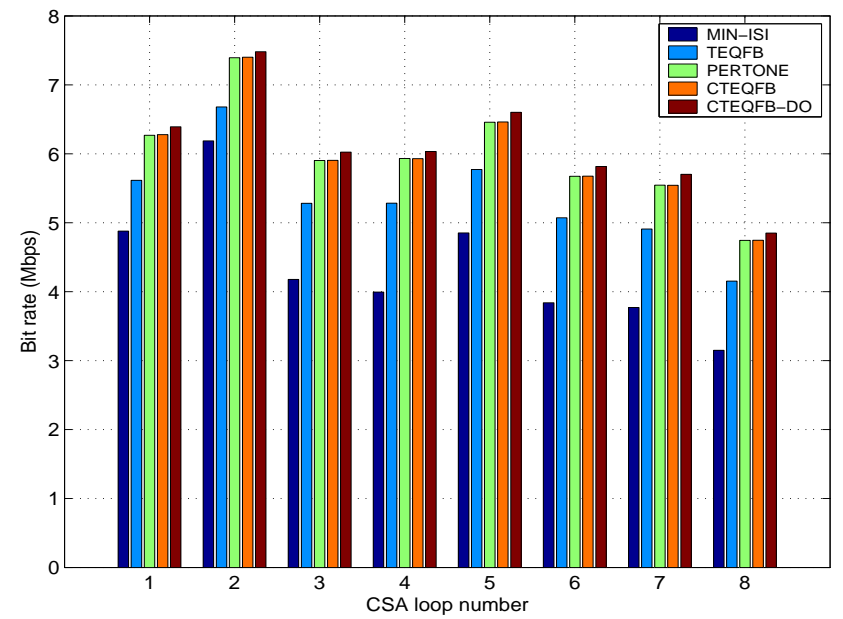

Figure 4: Achievable bit rates for $8 \mathrm{CSA}$ loops with length 17 equalizers. Coding gain is $5 \mathrm{~dB}$, margin is 6 $\mathrm{dB}$, input power is $-40 \mathrm{dBm} / \mathrm{Hz}$, AWGN power -140 $\mathrm{dBm} / \mathrm{Hz}, \mathrm{NEXT}$ noise is from 5 ISDN disturbers, RFI noise from 7 AM radio frequencies. Equalizer is trained by Min-ISI [5], bit rate maximizing TEQFB [9], least squares per-tone [11] and the proposed method.

QAM error energy over 1000 symbols

$$
\mathrm{SNR}_{i}=10 \log _{10}\left(\frac{1000 S_{x}}{\sum_{t=1}^{1000}\left|X_{t, i}-\hat{X}_{t, i}\right|^{2}}\right)
$$

and SNR gap is defined as

$$
\Gamma_{\text {sim }}(\text { in } \mathrm{dB})=\Gamma_{\text {gap }}+\text { system margin }- \text { coding gain }
$$

where $\Gamma_{\text {gap }}=9.8 \mathrm{~dB}$ corresponds to $10^{-7}$ bit error rate, system margin is $6 \mathrm{~dB}$, and coding gain is $5 \mathrm{~dB}$. The total number of bits per DMT symbol is $\sum_{i} b_{i}$.

Fig. 4 presents a summary of the results that we obtained for various equalizer designs. Min-ISI is chosen to optimize a conventional TEQ-FEQ structure, which is explained in detail in [5]. Least squares design (LS-PTE) is chosen for the per tone structure [8]. Bit rate maximizing TEQFB algorithm presented in [9] is used for TEQ filter bank structure. The training algorithm proposed in this paper is used for CTEQFB. The results of CTEQFB with and without delay optimization are both presented. Conventional TEQFEQ structure has much lower bit rate in this test setup that contains severe channel distortion and multiple impairments. Especially RFI noise creates deep notches in transmission band. BRM-TEQFB performance is somewhat lower than per-tone and CTEQFB due to its incomplete noise modelling. RFI, which is not included in TEQFB's SNR modelling, killed about
$10 \%$ bit rate when compared to LS-PTE or CTEQFB. LS-PTE and CTEQFB have equivalent performance. But if delay optimization is performed, CTEQFB has $2 \%$ performance improvement, which benchmarks the bit rate performance.

\section{REFERENCES}

[1] J. S. Chow, J. M. Cioffi, and J. A. C. Bingham, "Equalizer Training Algorithms for Multicarrier Modulation Systems," in Proc. IEEE Int. Conf. on Comm., May 1993, pp. 761-765.

[2] N. Al-Dhahir and J. M. Cioffi, "Efficiently Computed Reduced-Parameter Input-Aided MMSE Equalizers for ML Detection: A Unified Approach," IEEE Trans. on Info. Theory, vol. 42, no. 3, pp. 903-915, May 1996.

[3] P. J. W. Melsa, R. C. Younce, and C. E. Rohrs, "Impulse Response Shortening for Discrete Multitone Transceivers," IEEE Trans. on Comm., vol. 44, pp. 1662-1672, Dec. 1996.

[4] C. Yin and G. Yue, "Optimal Impulse Response Shortening for Discrete Multitone Transceivers," Electronics Letters, vol. 34, pp. 35-36, Jan. 1998.

[5] G. Arslan, B. L. Evans, and S. Kiaei, "Equalization for Discrete Multitone Receivers To Maximize Bit Rate," IEEE Trans. on Signal Processing, vol. 49, no. 12, pp. 3123-3135, Dec. 2001.

[6] M. Ding, B. L. Evans, R. K. Martin, and C. R. Johnson Jr., "Minimum Intersymbol Interference for Time Domain Equalizer Design," in Proc. IEEE GLOBECOM, Dec. 2003.

[7] M. Ding, A. J. Redfern, and B. L. Evans, "A Dualpath TEQ Structure For DMT-ADSL Systems," in Proc. IEEE Conf. on Acoustics, Speech, Signal Proc., May 2002, pp. 2573-2576.

[8] K. Van Acker, G. Leus, M. Moonen, O. van de Wiel, and T. Pollet, "Per Tone Equalization for DMT-Based Systems," IEEE Trans. on Comm., vol. 49, no. 1, pp. 109-119, Jan. 2001.

[9] M. Milosevic, L. F. C. Pessoa, B. L. Evans, and R. Baldick, "DMT Bit Rate Maximization With Optimal Time Domain Equalizer Filter Bank Architecture," in Proc. IEEE Asilomar Conf. on Signals, Systems, and Computers, Nov. 2002.

[10] N. Al-Dhahir and J. M. Cioffi, "Optimum FiniteLength Equalization for Multicarrier Transceivers," IEEE Trans. on Comm., vol. 44, no. 1, pp. 56-64, Jan. 1996.

[11] K. Vanbleu, G. Ysebaert, G. Cuypers, M. Moonen, and K. Van Acker, "Bitrate Maximizing Time-Domain Equalizer Design for DMT-based Systems," in Proc. IEEE Int. Conf. on Comm., May 2003, pp. 2360-2364.

[12] G. Arslan, M. Ding, B. Lu, M. Milosevic, Z. Shen, and B. L. Evans, "Multicarrier Equalization Design Toolbox," The University of Texas at Austin, www.ece.utexas.edu/ bevans/projects/adsl/dmtteq. 